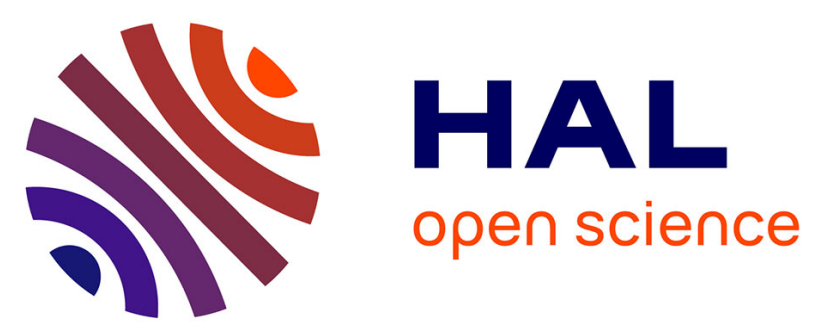

\title{
An ISO10218-compliant adaptive damping controller for safe Physical Human-Robot Interaction
}

Benjamin Navarro, Andrea Cherubini, Aïcha Fonte, Robin Passama, Gérard Poisson, Philippe Fraisse

\section{- To cite this version:}

Benjamin Navarro, Andrea Cherubini, Aïcha Fonte, Robin Passama, Gérard Poisson, et al.. An ISO10218-compliant adaptive damping controller for safe Physical Human-Robot Interaction. ICRA: International Conference on Robotics and Automation, May 2016, Stockholm, Sweden. pp.3043-3048, 10.1109/ICRA.2016.7487468 . hal-01274736

\section{HAL Id: hal-01274736 https://hal.science/hal-01274736}

Submitted on 16 Feb 2016

HAL is a multi-disciplinary open access archive for the deposit and dissemination of scientific research documents, whether they are published or not. The documents may come from teaching and research institutions in France or abroad, or from public or private research centers.
L'archive ouverte pluridisciplinaire HAL, est destinée au dépôt et à la diffusion de documents scientifiques de niveau recherche, publiés ou non, émanant des établissements d'enseignement et de recherche français ou étrangers, des laboratoires publics ou privés. 


\title{
An ISO10218-compliant adaptive damping controller for safe Physical Human-Robot Interaction
}

\author{
Benjamin Navarro ${ }^{1,2}$, Andrea Cherubini ${ }^{1}$, Aïcha Fonte ${ }^{2}$, Robin Passama ${ }^{1}$, Gérard Poisson ${ }^{2}$, and Philippe Fraisse ${ }^{1}$
}

\begin{abstract}
In human-robot interaction, the robot must behave safely, especially when an operator is present in its workspace. Even higher safety levels must be attained when physical contact occurs between the two. To this end, standards such as the ISO10218 define the requirements for a robot to be considered safe for interaction with human operators in an industrial environment. In this paper, we propose an adaptive damping controller that fulfills the ISO10218 requirements by limiting the tool velocity, power and contact force online (and only when needed). The controller is experimentally validated on a hand-arm robotic system, in a mock-up collaborative application. For the hand, safe interaction is enhanced by using tactile sensing, both to regulate grasp forces and to provide an intuitive interface for the operator.
\end{abstract}

\section{INTRODUCTION}

In recent years, a growth of interest for physical human robot interaction (pHRI) has emerged in robotics research [1], [2], [3]. The goal is to enable collaborative working between human and robots, since Cobots (Collaborative Robots) can improve the flexibility of industrial processes, while decreasing the operators' fatigue [4].

In such scenarios, the robot must adopt a safe behaviour to minimize the risk of injuries to close coworkers. However, until recently, precise requirements for a collaborative robot were not specified. In 2011, in the last revision of the ISO10218 standard [5], the International Organization for Standardization included requirements for a safe industrial robot. This standard specifies that any robot must respect velocity, power and contact force limits at the tool control point (TCP) in the presence of a human. These three limits are fixed by the end-user, depending on the performed task and on the degree of collaboration between operator and robot.

To our knowledge, present-day collaborative robot manufacturers [6] fulfill the standard by saturating the velocity, stopping the robot, or by using expensive hardware solutions. Although novel safe actuation systems have been recently proposed in the literature [7], [8], [9], these are not always easily affordable or adaptable for any robotic system.

An alternative comes from control, typically impedance control [10], and its modified versions for force tracking [11], force limitation [12], adaptive damping [13] or exploiting redundancy [14]. However, to the best of our knowledge, the only work that explicitly tackles the ISO1028-2011 from

\footnotetext{
${ }^{1}$ Laboratory for Computer Science, Microelectronics and Robotics LIRMM - Université de Montpellier CNRS, 161 Rue Ada, 34090 Montpellier, France. E-mail: firstname.lastname@lirmm.fr

${ }^{2}$ PRISME Laboratory, University of Orléans - INSA CVL, 63 avenue de Lattre de Tassigny, F-18020 Bourges Cedex, France. E-mail: firstname.lastname@univ-orleans.fr
}

a control viewpoint is [15]. Nevertheless, this controller only takes into account the force limitation imposed by the standard.

In the frame of the SISCob project, we propose an impedance (specifically, a damping) controller that automatically adapts to the external wrench, to guarantee that all three ISO10218 limitations (force, velocity, and power) are respected. The controller has been validated on a robotic arm, with a hand mounted on the end-effector.

The paper is organized as follows. In Section II, we define the main variables. Sections III and IV respectively describe the adaptive damping controller, and the complete framework that we design to respect force, velocity, and power limitations. The experimental validation on a robotic hand-arm system in a mock-up collaborative scenario is presented in Section V. In this section, we also explain the use of tactile sensing, both to regulate the grasp and to provide an intuitive HRI interface. We summarize and conclude in Section VI.

\section{DEFINITIONS}

We consider a robot manipulator (open kinematic chain) with $j$ degrees of freedom (dof), and denote: $\mathbf{q} \in \mathbb{R}^{j}$ its joint values, $\mathbf{x} \in \mathbb{S E}(3)$ the TCP pose, and $\dot{\mathbf{x}}=\left[\mathbf{v}^{\top} \boldsymbol{\omega}^{\top}\right]^{\top}$ the TCP kinematic screw. Both $\mathbf{x}$ and $\dot{\mathbf{x}}$ are expressed in the robot base frame. We also assume that it is possible to estimate (either through direct or through joint torque measurement) the external wrench (force and torque) applied to the TCP, and expressed in the TCP frame: $\mathbf{h}=\left[\mathbf{f}^{\top} \mathbf{m}^{\top}\right]^{\top} \in \mathbb{R}^{6}$. This information will be used to adapt the robot motion to make it compliant, while respecting the ISO1028-2011 standard. To calculate the power $P$ transmitted by the robot, the wrench must be expressed in the base frame, as ${ }^{B} \mathbf{W}_{T} \mathbf{h}$, where:

$$
{ }^{B} \mathbf{W}_{T}=\left[\begin{array}{cc}
{ }^{B} \mathbf{R}_{T} & \mathbf{0}_{3} \\
\mathbf{0}_{3} & { }^{B} \mathbf{R}_{T}
\end{array}\right],
$$

with ${ }^{B} \mathbf{R}_{T}$ the rotation matrix between the TCP and the base frame. Given the above, we express the standard by ${ }^{1}$ :

$$
\begin{gathered}
|\mathbf{f}| \leq F_{M} \\
P=\left\langle{ }^{B} \mathbf{W}_{T} \mathbf{h}, \dot{\mathbf{x}}\right\rangle \leq P_{M},
\end{gathered}
$$$$
|\mathbf{v}| \leq V_{M}
$$

with positive scalars $F_{M} \in \mathbb{R}_{+}^{*}$ the maximum external force allowed, $V_{M} \in \mathbb{R}_{+}^{*}$ the maximum velocity allowed, and $P_{M} \in \mathbb{R}_{+}^{*}$ the maximum transmitted power allowed.

\footnotetext{
${ }^{1}$ Throughout the paper, we denote by $|\mathbf{u}|$ the Euclidean norm of vector $\mathbf{u}$, and $\langle\mathbf{u}, \mathbf{v}\rangle$ the dot product of vectors $\mathbf{u}$ and $\mathbf{v}$.
} 
The contribution of this paper will be the development of a feedback controller, which, based on the measure of $\mathbf{h}$, will design $\dot{\mathbf{x}}$ to comply with constraints (2)-(4).

In our framework, inverse kinematics is used to track the desired tool pose, $\mathbf{x}^{*}$. This pose can be any subset of $\mathbb{S E}(3)$, provided that it is reachable, with the robot kinematics and dof. Then, the evolution of pose $\mathbf{x}$ can be expressed as:

$$
\dot{\mathbf{x}}=\mathbf{J} \dot{\mathbf{q}}
$$

with $\mathbf{J}=\frac{\partial \mathbf{x}}{\partial \mathbf{q}}$ the task Jacobian, that is derived from the robot measured configuration $\hat{\mathbf{q}}$. The tool pose can be regulated from the current value $\mathbf{x}$ to a desired value $\mathbf{x}^{*}$, by applying the joint velocity:

$$
\dot{\mathbf{q}}=\mathbf{J}^{\dagger} \lambda\left(\mathbf{x}^{*}-\mathbf{x}\right) .
$$

In the above equation: $\mathbf{J}^{\dagger}$ is the right pseudoinverse ${ }^{2}$ of $\mathbf{J}$, and $\lambda$ is a positive scalar gain. It is well known that system (5), controlled by (6), is globally asymptotically stable with respect to the pose regulation task. Indeed, plugging (6) into (5) yields:

$$
\dot{\mathbf{x}}=\lambda\left(\mathbf{x}^{*}-\mathbf{x}\right)
$$

and, since $\lambda>0, \mathbf{x}=\mathbf{x}^{*}$ is a stable equilibrium for the closed-loop system. In practice, if we neglect the dynamic effects, the robot reacts as a first order system, with time constant $\tau=\frac{1}{\lambda}$, and response time:

$$
t_{95 \%}=3 \tau=3 / \lambda .
$$

This will be used to account for the robot response delay.

\section{ADAPTIVE DAMPING CONTROL}

To adapt the desired pose of the tool center in the presence of external forces, we use impedance control [10], only with damping. This consists in applying a tool velocity which, expressed in the tool frame, is linear with the applied wrench:

$$
{ }^{T} \dot{\mathbf{x}}_{i m p}=\mathbf{B}^{-1} \mathbf{h}
$$

with $\mathbf{B}=\left[\begin{array}{cc}\mathbf{B}_{t} & \mathbf{0}_{3} \\ \mathbf{0}_{3} & \mathbf{B}_{r}\end{array}\right]=\operatorname{diag}\left\{b_{x}, b_{y}, b_{z}, b_{r x}, b_{r y}, b_{r z}\right\} \quad$ a diagonal matrix of strictly positive and constant damping gains. These are pre-tuned by the operator, depending on the damping effect desired in each direction. With this strategy, the tool will move away from the applied wrench, facilitating the realization of (2). On the other hand, this can lead to cases where either the applied velocity or the transmitted power pass the limits imposed by (3) and (4). To avoid infringing these constraints, we include in the damping controller an adaptive gain $K \leq 1$ :

$$
{ }^{T} \dot{\mathbf{x}}_{i m p}=K \mathbf{B}^{-1} \mathbf{h} .
$$

To avoid breaking (3) (respectively (4)), $K$ is decreased to the values $K_{v}<1$ (respectively $K_{p}<1$ ), leading to higher damping, hence slowing the robot. In the next subsections, we will explain how the values of $K_{v}$ and $K_{p}$ are calculated.

The velocity induced by (9) must be mapped from the tool to the base frame to be realizable by the inverse kinematics

\footnotetext{
${ }^{2}$ We assume that $\mathbf{J}$ is full row rank during operation, so that this pseudoinverse can be calculated. This is a common assumption in inverse kinematics control.
}

controller (6). To this end, it must be premultiplied by the spatial motion transform matrix from tool to base frame:

$$
\dot{\mathbf{x}}_{i m p}={ }^{B} \mathbf{V}_{T}{ }^{T} \dot{\mathbf{x}}_{i m p}=\left[\begin{array}{cc}
{ }^{B} \mathbf{R}_{T} & \mathbf{0}_{3} \\
\mathbf{0}_{3} & { }^{B} \mathbf{R}_{T}
\end{array}{ }^{T} \dot{\mathbf{x}}_{i m p} .\right.
$$

Furthermore, we must take into account the reference trajectory demanded to the robot (e.g., defined by an offline motion planner), and denoted by $\dot{\mathbf{x}}_{r}=\left[\begin{array}{ll}\mathbf{v}_{r}^{\top} & \boldsymbol{\omega}_{r}^{\top}\end{array}\right]^{\top}$. The desired trajectory should be adapted from $\dot{\mathbf{x}}_{r}$ in the presence of external wrench:

$$
\dot{\mathbf{x}}=\dot{\mathbf{x}}_{i m p}+\dot{\mathbf{x}}_{r}
$$

and must be designed in accordance with the ISO standard:

$$
\left|\mathbf{v}_{r}\right| \leq V_{M}
$$

In summary, the relationship between the external wrench $\mathbf{h}$ and the tool velocity $\dot{\mathbf{x}}$ in the base frame is:

$$
\dot{\mathbf{x}}=K^{B} \mathbf{V}_{T} \mathbf{B}^{-1} \mathbf{h}+\dot{\mathbf{x}}_{r} .
$$

Since the ISO standard focuses uniquely on the translation components of force and velocity, we consider only the first three lines of the above equation:

$$
\mathbf{v}=K \mathbf{Y} \mathbf{f}+\mathbf{v}_{r} .
$$

The $3 \times 3$ matrix $\mathbf{Y}$ is the controller translation admittance:

$$
\mathbf{Y}={ }^{B} \mathbf{R}_{T} \mathbf{B}_{t}^{-1} \text {. }
$$

In the remainder of this section, we will explain how the values of $K$ in (15) are adapted to fulfill constraints (2)-(4). We assume that $\mathbf{f} \neq \mathbf{0}$, since otherwise, $\mathbf{v} \leq \mathbf{v}_{r}$ fulfills the constraints. If we assume non-infinite damping gains we can state that $\mathbf{f} \notin \operatorname{ker} \mathbf{Y}$ since $\operatorname{ker}{ }^{B} \mathbf{R}_{T}=\mathbf{0}$.

\section{A. Dealing with the force limitation}

Let us first consider (2). The ISO10218 standard limits the force exchanged between tool and environment. However, in the case of interaction with a human (e.g., in case of collision), it is very difficult to model/predict the evolution of such force over time. Besides, since we consider the robot to be intrinsically a delayed closed-loop system (as shown in Sect. II), our controller will employ some time to dampen the force. We can, however, assume that the force derivative is bounded:

$$
|\dot{\mathbf{f}}| \leq \dot{F}_{M}
$$

This is a reasonable hypothesis when considering physical interaction with humans (excluding collisions). Furthermore, the value of $\dot{F}_{M}$ can be determined experimentally, by measuring the forces exerted by the human during typical operation. Then, using (17) and (8), we can determine the maximum increment of the force norm that could occur while the system responds:

$$
\Delta F_{M}=\dot{F}_{M} t_{95 \%}=3 \dot{F}_{M} / \lambda .
$$

The strategy that we propose in this scope consists in moving the robot as quickly as possible away from the contact, without breaking the velocity or the power limit. This is equivalent to applying a high $K$ in (15) if the external force is above the security threshold $F_{M}-\Delta F_{M}$. 
In summary, to comply with the ISO constraints (3) and (4), we set $K$ to:

$$
K= \begin{cases}\min \left\{K_{v}, K_{p}, 1\right\} & \text { if }|\mathbf{f}| \leq F_{M}-\Delta F_{M}, \\ \min \left\{K_{v}, K_{p}\right\} & \text { otherwise. }\end{cases}
$$

Note that in both cases, $K$ is chosen conservatively to guarantee that neither the velocity (since $K \leq K_{v}$ ) nor the power (since $K \leq K_{p}$ ) constraints are infringed. Moreover, if the force is below the limit, the damping will stay above its pre-tuned values (because $K \leq 1$ ), whereas otherwise, it could go below these values, to move the robot away from collision. In the next two subsections, we detail the calculations of $K_{v}$ and $K_{p}$, respectively.

\section{B. Dealing with the velocity limitation}

Let us now consider the velocity limitation (3). Squaring that equation, and using (15), along with Pythagoras' theorem, yields:

$$
K^{2}|\mathbf{Y} \mathbf{f}|^{2}+2 K\left\langle\mathbf{Y} \mathbf{f}, \mathbf{v}_{r}\right\rangle+\left|\mathbf{v}_{r}\right|^{2}-V_{M}^{2} \leq 0,
$$

This quadratic equation is verified for:

$$
\begin{aligned}
& K \in\left[\frac{-\left\langle\mathbf{Y} \mathbf{f}, \mathbf{v}_{r}\right\rangle-\sqrt{\left\langle\mathbf{Y} \mathbf{f}, \mathbf{v}_{r}\right\rangle^{2}-|\mathbf{Y} \mathbf{f}|^{2}\left(\left|\mathbf{v}_{r}\right|^{2}-V_{M}^{2}\right)}}{|\mathbf{Y}|^{2}},\right. \\
&\left.\frac{-\left\langle\mathbf{Y} \mathbf{f}, \mathbf{v}_{r}\right\rangle+\sqrt{\left\langle\mathbf{Y} \mathbf{f}, \mathbf{v}_{r}\right\rangle^{2}-|\mathbf{Y} \mathbf{f}|^{2}\left(\left|\mathbf{v}_{r}\right|^{2}-V_{M}^{2}\right)}}{|\mathbf{Y} \mathbf{f}|^{2}}\right] .
\end{aligned}
$$

If interval (21) includes 1 , the velocity constraint is guaranteed by the pre-tuned damping values $(K=1)$. Otherwise, we should apply the upper bound to stay as near as possible to the pre-tuned damping while guaranteeing (3). This corresponds to:

$$
K_{v}=\min \left\{\frac{-\left\langle\mathbf{Y} \mathbf{f}, \mathbf{v}_{r}\right\rangle+\sqrt{\left\langle\mathbf{Y} \mathbf{f}, \mathbf{v}_{r}\right\rangle^{2}-|\mathbf{Y} \mathbf{f}|^{2}\left(\left|\mathbf{v}_{r}\right|^{2}-V_{M}^{2}\right)}}{|\mathbf{Y} \mathbf{f}|^{2}}\right.
$$

It can be shown easily from (22) that $K_{v}$ can become 0 when the external force tends to align with the reference velocity while $\left|\mathbf{v}_{r}\right|=V_{M}$. This means that the velocity can no longer be increased by the operator in order to respect (3).

\section{Dealing with the power limitation}

Let us finally consider the power limitation (4). This can be rewritten, using (14), as:

$$
\left\langle{ }^{B} \mathbf{W}_{T} \mathbf{h}, K^{B} \mathbf{V}_{T} \mathbf{B}^{-1} \mathbf{h}+\dot{\mathbf{x}}_{r}\right\rangle \leq P_{M} .
$$

However, the wrench may vary while the system responds. We deal with this, as in Sect. III-A, by assuming that its derivative is bounded:

$$
\left|\frac{d}{d t}\left({ }^{B} \mathbf{W}_{T} \mathbf{h}\right)\right| \leq \dot{H}_{M} .
$$

Then, using (8), we can determine the maximum relative increment of the wrench norm (in the base frame) that could occur while the system responds:

$$
\Delta H_{M \%}=\frac{\dot{H}_{M}}{\left|{ }^{B} \mathbf{W}_{T} \mathbf{h}\right|} t_{95 \%}=\frac{3 \dot{H}_{M}}{\left.\lambda\right|^{B} \mathbf{W}_{T} \mathbf{h} \mid} .
$$

This expression is well defined since $\operatorname{ker}{ }^{B} \mathbf{W}_{T}=\mathbf{0}$, and it corresponds to a variation of the transmitted power to be taken into account in the security constraint:

$$
\left\langle\left(1+\Delta H_{M \%}\right)^{B} \mathbf{W}_{T} \mathbf{h}, K^{B} \mathbf{V}_{T} \mathbf{B}^{-1} \mathbf{h}+\dot{\mathbf{x}}_{r}\right\rangle \leq P_{M} .
$$

Then, since ${ }^{B} \mathbf{W}_{T}^{\top}={ }^{B} \mathbf{V}_{T}^{-1}$, (4) is guaranteed if:

$$
\left.K \in]-\infty, \frac{P_{M} /\left(1+\Delta H_{M \%}\right)-\mathbf{h}^{\top T} \mathbf{V}_{B} \dot{\mathbf{x}}_{r}}{\mathbf{h}^{\top} \mathbf{B}^{-1} \mathbf{h}}\right] .
$$

Since $\mathbf{B}^{-1}$ is positive definite, (27) is well defined. If interval (27) includes 1 , the power constraint is guaranteed by the pre-tuned damping values $(K=1)$. Otherwise, we should apply the upper bound to stay as near as possible to the pretuned damping, while guaranteeing (4). This corresponds to:

$$
K_{p}=\min \left\{\frac{P_{M} /\left(1+\Delta H_{M \%}\right)-\mathbf{h}^{\top} \mathbf{V}_{B} \dot{\mathbf{x}}_{r}}{\mathbf{h}^{\top} \mathbf{B}^{-1} \mathbf{h}}, 1\right\} .
$$

It can be seen that an increase in $\mathbf{h}$ will require lower $K_{p}$, i.e., higher damping. The value of $K_{p}$ can become negative only by effect of the term $\mathbf{h}^{\top T} \mathbf{V}_{B} \dot{\mathbf{x}}_{r}$. This term can grow, e.g., when the external wrench tends to align with the reference kinematic screw, and both are important. Then, the robot has to oppose the external force (instead of complying with it) by reducing its velocity to avoid infringing (4).

\section{CONTROL FRAMEWORK}

Let us hereby present our proposed framework for safe pHRI, that is illustrated in Fig. 1. At each iteration, the tool reference pose is modified by recursively integrating (14): $\mathbf{x}^{*}(t)=\mathbf{x}_{r}(t)+\Delta \mathbf{x}(t)$. Pose variation $\Delta \mathbf{x}$ is calculated by taking the first order finite difference approximation of (14) over a sampling period $T_{s}$ :

$\Delta \mathbf{x}(t)= \begin{cases}0 & \text { if } t<T_{s}, \\ \Delta \mathbf{x}\left(t-T_{s}\right)+\left(K^{B} \mathbf{V}_{T} \mathbf{B}^{-1} \mathbf{h}(t)+\dot{\mathbf{x}}_{r}(t)\right) T_{s} & \text { otherwise. }\end{cases}$

In this equation, $K$ is adapted to the working conditions, according to (19). It can be set to either $1, K_{v}$ or $K_{p}$, with $K_{v}$ and $K_{p}$ defined respectively in (22) and (28). Then, $\mathbf{x}^{*}$ is tracked by inverse kinematics (6). For a position-controlled robot, we need to recursively integrate that equation, to compute the target joint position:

$$
\mathbf{q}^{*}(t)= \begin{cases}\mathbf{q}(0) & \text { if } t<T_{s}, \\ \mathbf{q}^{*}\left(t-T_{s}\right)+\mathbf{J}^{\dagger} \lambda T_{s}\left(\mathbf{x}^{*}-\mathbf{x}\right) & \text { otherwise. }\end{cases}
$$

In summary, the proposed framework for safe pHRI is based on (19), (29), and (30), as outlined in Fig. 1. This framework enables the operator to manipulate the robot safely without the risk of exceeding the velocity, power and force limitations imposed by the application. 


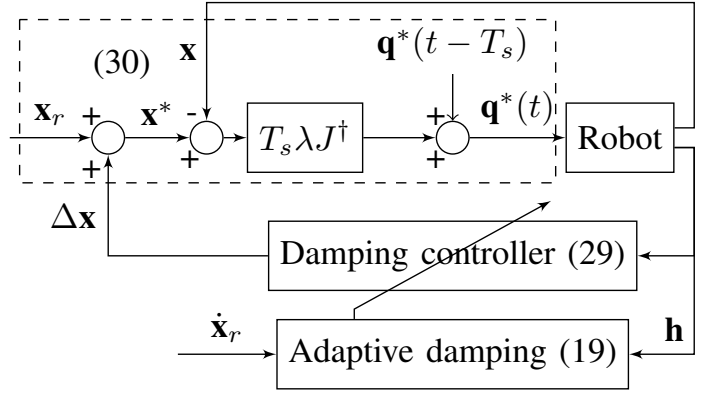

Fig. 1: Outline of the proposed safe control framework

\section{REAL EXPERIMENTS}

\section{A. Setup}

To validate our control framework, we focus on an industrial screwing application (see Fig. 3). An operator manipulates a robotic hand-arm system to grasp an electric screwdriver, move it to a desired position, and then perform the screwing. The system is composed of a Kuka LWR4 arm, with a Shadow Right Hand mounted on its end effector. The five hand fingertips are each equipped with a tactile sensor (Syntouch BioTac), while the external wrench $\mathbf{h}$ on the Kuka effector is estimated through the FRI Interface ${ }^{3}$. This wrench also includes the dynamic effects applied to the TCP (hand inertia, Coriolis forces, friction, etc), excluding gravitation. In the experiment, the proposed ISO10218-based control framework is applied to the Kuka end effector (corresponding to the hand wrist). The main reason for this is that the BioTacs provide only force (not torque) measurement, and solely on one side of the fingertip. Therefore, a wrench estimator should be designed in order to extend (14) to the hand fingertips. This estimator will be the object of future work. Nevertheless, our choice is justified by the fact that: 1) the operator manipulates the system at the Kuka end effector, which can therefore be considered as the tool in this application, and 2) given their mechanical design and characteristics, the Shadow Hands are intrinsically safe (they are specifically conceived for pHRI).

Although we did not apply our control framework to the hand, we did guarantee safety of its use, by exploiting tactile data in two ways. First, we designed a simple grasp strategy that is driven by tactile measures. Secondly, we exploited the thumb BioTac as an interface for intuitive human-robot interaction. These two aspects will be detailed just below.

\section{B. Safe hand control}

In this section, we describe the implemented safe hand controller. We will refer only to one finger, since the methods can be extended, without loss of generality, to all $n$ fingers. The proposed control is feasible on any hand with $n \geq 4$ : at least 3 fingers for grasping, and 1 for intuitive interaction with the operator. As in [16], we do not prove the stability of our grasp. However the grasp will regulate the magnitude of the fingertip contact forces. The estimation of these magnitudes $\mathbf{F}$ using BioTac sensors has been done in our previous work [17] which was inspired by [18]. In fact, by determining experimentally the values $\mathbf{F}^{*}>0$ needed to firmly grasp an object, we can use these as target control values. The

\footnotetext{
${ }^{3}$ http://cs.stanford.edu/people/tkr/fri/html
}

fingers are controlled in the articular space $\mathbf{q}$, with an online trajectory generator, noted OTG. This OTG will generate a smooth trajectory between the current articular configuration, and a desired one $\mathbf{q}^{*}$, under velocity constraint $\dot{\mathbf{q}}_{M} \geq 0$. We will modify online the OTG inputs to regulate the contact forces between finger and object. Depending on $\mathbf{F}$, we can choose between going to the closed finger position, $\mathbf{q}^{c}$, or back to the opened finger position, $\mathbf{q}^{o}$. Both $\mathbf{q}^{c}$ are $\mathbf{q}^{o}$ are determined experimentally in offline tests, and depend on the grasped object's shape and size. The OTG inputs for finger $i$ are set to:

$$
\begin{aligned}
\mathbf{q}_{i}^{*}(t) & = \begin{cases}\mathbf{q}_{i}^{c} & \text { if } F_{i}<F_{i}^{*} \\
\mathbf{q}_{i}^{o} & \text { otherwise, }\end{cases} \\
\dot{\mathbf{q}}_{M i} & = \begin{cases}\dot{\mathbf{q}}_{M i}^{0} & \text { if } F_{i}>2 F_{i}^{*} \\
\frac{\left|F_{i}^{*}-F_{i}\right|}{F_{i}^{*}} \dot{\mathbf{q}}_{M i}^{0} & \text { otherwise. }\end{cases}
\end{aligned}
$$

Vector $\dot{\mathbf{q}}_{M i}^{0}$ is the default finger joint velocity limit (as provided in the hand datasheet). In practice, the finger velocity is scaled in the presence of a contact force $\left.F_{i} \in\right] 0,2 F_{i}^{*}[$, and it opens or closes depending on its value wrt $F_{i}^{*}$. With all parameters defined, we can use the OTG to drive the finger:

$$
\mathbf{q}_{i}(t)= \begin{cases}\operatorname{OTG}\left(\mathbf{q}_{i}\left(t-T_{s}\right), \mathbf{q}_{i}^{*}, \dot{\mathbf{q}}_{M i}\right) & \text { if }\left|F_{i}^{*}-F_{i}\right|>\epsilon \\ \mathbf{q}_{i}\left(t-T_{s}\right) & \text { otherwise, }\end{cases}
$$

with $\epsilon>0$ a small value avoiding oscillations around the target value, once $F_{i}^{*}$ is attained.

If $n>3$, one of the $n$ tactile sensors can be used as a button to trigger some events (e.g., to start the grasping). The implemented switch is based on a comparator with hysteresis:

$$
S(t)= \begin{cases}1 & \text { if }|F|>F_{H} \\ 0 & \text { if }|F|<F_{L} \\ S\left(t-T_{s}\right) & \text { otherwise }\end{cases}
$$

where $F_{H}$ and $F_{L}\left(F_{H}>F_{L}>0\right)$ are the pre-tuned high and low thresholds at which the state changes. With this triggering system, the operator can command the robot during interaction without the need of an external, sophisticated interface. This solution improves both the ergonomy and the time required to perform the task.

\section{Results}

The implementation has been realized on a computer with an i7-4600U processor running Linux. All the code was written in C++ using the Knowbotics framework, currently developed at LIRMM $^{4}$. The FRI was used to communicate with the Kuka arm, and a ROS interface ${ }^{5}$ to control the Shadow Hand and get the BioTacs measures. Kinematic computations were performed using the Robotics Library ${ }^{6}$. The controller sample time was $T_{s}=5 \mathrm{~ms}$ and the average computation time required for each loop was $0.3 \mathrm{~ms}$. To generate the arm reference trajectory $\dot{\mathbf{x}}_{r}$, and for the hand OTG, we use the Reflexxes library ${ }^{7}$.

\footnotetext{
${ }^{4}$ https://gite.lirmm.fr

${ }^{5}$ http://wiki.ros.org/shadow_robot

${ }^{6}$ http: / / www.roboticslibrary.org

${ }^{7}$ www.reflexxes.ws
} 
To handle the various steps required by our application, we design the state machine shown in Fig. 2 (the term tool refers to the arm end-effector, where the hand is mounted and where the ISO10218 standard is guaranteed). Boxes represent autonomous (or semi-autonomous) actions performed by the robot, ellipses collaborative operations, and diamonds are tests. Arrows with an 'S' are triggered by the tactile switch (the thumb is used), on the falling edge of $S$ in (34) (we use $F_{H}=3 \mathrm{~N}$ and $F_{L}=0.5 \mathrm{~N}$ ).

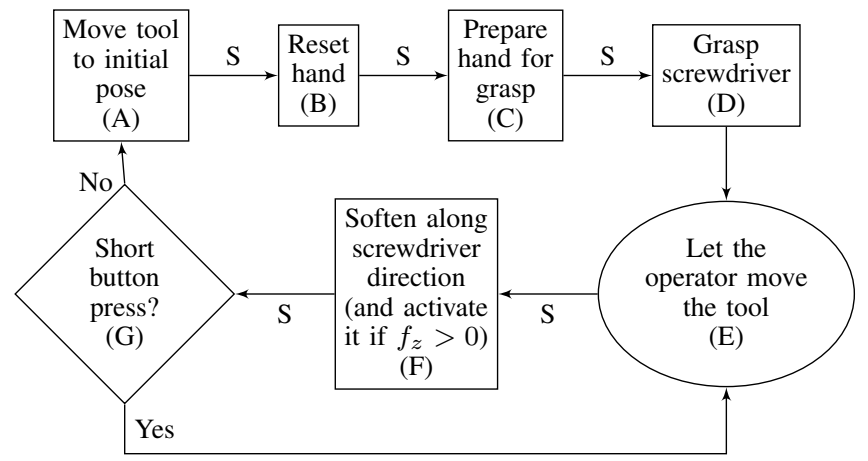

Fig. 2: State machine for collaborative screwing.

We set $\lambda=1$ when $\dot{\mathbf{x}}_{r}$ varies (state (A)), and $\lambda=5$ when $\dot{\mathbf{x}}_{r}=\mathbf{0}$ (all other states). The damping is set to $\mathbf{B}=\operatorname{diag}\{250,250,250,20,20,20\}$, except in state $(\mathrm{F}) . \mathrm{We}$ hereby detail the state machine.

(A) The tool moves to an initial fixed pose $\mathbf{x}_{r}^{*}$, while the hand is still.

(B) The tool is still, while the hand is reset to its default configuration.

(C) The tool is still, while the hand prepares for grasping (each finger is driven to its $\mathbf{q}^{o}$ ). The operator hands the screwdriver to the robot before triggering (D).

(D) The tool is still, while the hand grasps the screwdriver, as explained in Sect.V-B. We set: $F^{*}=3 \mathrm{~N}$ for the forefinger and ring finger, $F^{*}=2 \mathrm{~N}$ for the little finger, and $\epsilon=0.01 \mathrm{~N}$.

(E) The operator can translate and reorient the tool using (14), with $\dot{\mathbf{x}}_{r}^{*}=\mathbf{0}$. When the desired screwing position is reached, the operator triggers $(\mathrm{F})$.

(F) Tool motion is tolerated only along the screwdriver direction ( $z$ axis of the tool frame), by setting $\mathbf{B}=\operatorname{diag}\left\{10^{5}, 10^{5}, 250,10^{5}, 10^{5}, 10^{5}\right\}$. If the operator pushes the tool forward, the middle finger powers the screwdriver. Otherwise, the screwdriver is stopped. For this, the middle finger is controlled in open-loop, since its BioTac does not touch the screwdriver switch.

(G) The operator can choose (via the thumb pressure duration) between pursuing screwing or making the tool return to its initial position.

Throughout the experiment, all changes in the external wrench are handled by our safe damping controller (14), with $K$ adapted through (19). We set: $V_{M}=0.2 \mathrm{~m} . \mathrm{s}^{-1}$, $P_{M}=20 \mathrm{~W}, F_{M}=150 \mathrm{~N}$, and $\dot{F}_{M}=\dot{H}_{M}=40 \mathrm{~N} / \mathrm{s}$.

A typical experiment is shown in the video attached to this paper $^{8}$, with snapshots in Fig. 3. During the experiment, two screwing operations at different positions are performed by

${ }^{8}$ See also: https://youtu.be/iOuhFKp31xY the operator (i.e., states $(\mathrm{E})$ and $(\mathrm{F})$ are repeated twice). In Fig. 4, we plot the evolution of $K,|\mathbf{f}|,|\mathbf{v}|,|\mathbf{P}|$ and of the four fingertip contact forces (all except the middle finger) during the various states. The curves show that by modulating $K$, the robot successfully respects all three limitations during the whole experiment. For example, at $t \simeq 15 \mathrm{~s}$, the tool velocity is reduced ( $K_{v}$ is applied, green area), and successively ( $t \simeq 16 \mathrm{~s}), K_{p}$ is applied (red area), to maintain the power under the maximum value allowed. Then, $K_{v}$ is applied again. On the other hand, during the two screwing phases $(F)$, the interaction forces remain too low to become critical. Thus, the initial damping values are maintained $(K=1)$. Although the value of $K$ changes eight times throughout the experiment, between $K_{p}, K_{v}$ and 1 , its trend is always smooth, making the robot motion natural. It is also important to notice that even with the perturbation due to the actuation of the screwdriver ( $t \simeq 20 \mathrm{~s}$ and $t \simeq 28 \mathrm{~s}$ ), contact forces at the fingertips (bottom curves) track their target values $\left(F^{*}=3 \mathrm{~N}\right.$ and $\left.F^{*}=2 \mathrm{~N}\right)$. This regulation guarantees a correct grasp throughout the experiment. Also note that the use of the thumb tactile sensor (cyan curve) as humanmachine interface permits completion of all operations by a single, untrained operator in an intuitive way, without the need of an external interface.

In the current setup, it was not possible to check the controller's behavior in case of sudden collisions, since the Kuka low-level security (which runs at $1 \mathrm{~ms}$, i.e., five times faster than our controller) deactivated the motors before adaptive damping could intervene. Solving this technical issue, to make the control loop run at $1000 \mathrm{~Hz}$, will be the object of future work. We are confident that sudden collisions can be managed, since even in the presence of force variations greater than $\dot{F}_{M}$ (e.g., up to $250 \mathrm{~N} / \mathrm{s}$ at $t \simeq 23$ s, see Fig. 4 ), the controller guaranteed the ISO limits.

\section{CONCLUSION}

The focus of this paper is the safety of physical interaction with a hand-arm robotic system.

The main contribution is the design of a closed-loop adaptive damping controller guaranteeing the ISO10218 standard (force, velocity and power limitations). We discuss some interesting aspects that emerge from our approach, but that should be considered whenever dealing with the standard, without dedicated hardware (as in [7], [8], [9]). First, the delay in the system response can cause the controller to infringe the ISO10218 when the force varies suddenly. For this, hardware solutions can come in handy but are not always available. Secondly, in some situations, the robot should (surprisingly!) become active instead of compliant. This is the case, e.g., when the force direction is aligned with a strong pre-planned velocity. These two aspects should be tackled with advanced models of the force evolution (e.g., based on model predictive control), which are non-trivial, particularly in the case of deformable contacts. Another contribution of our work is the use of tactile sensor as an intuitive human-robot interface for safe hand control.

In future work, we plan to extend the adaptive damping controller to the robotic hand and/or to humanoids and to a complete impedance model (mass-damping-stiffness). 


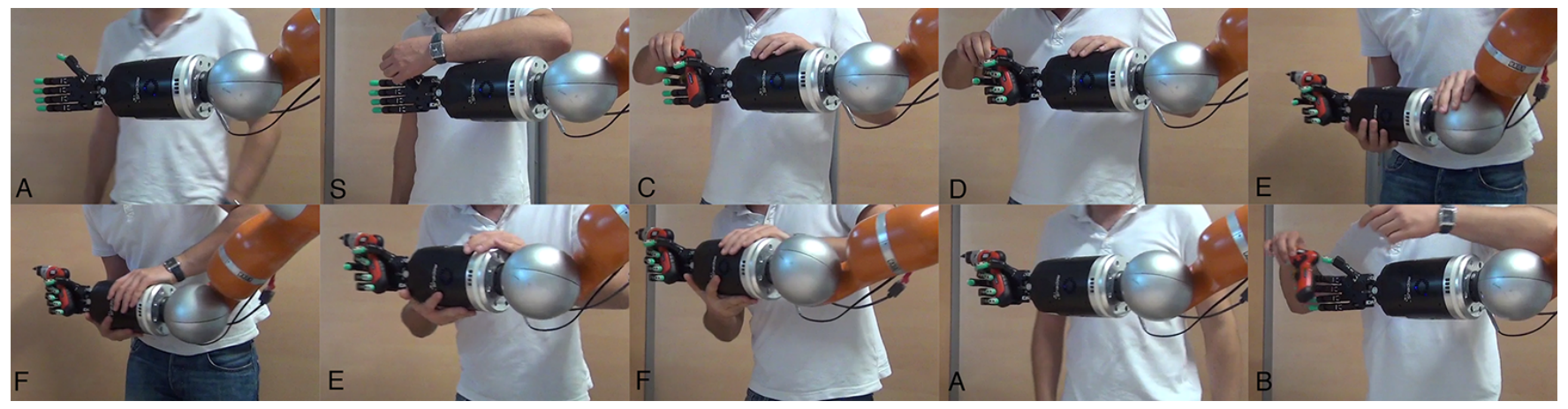

Fig. 3: Snapshots of the collaborative screwing experiment. Current state (left to right, top to bottom): (A) going to initial pose, (S) pushing thumb switch, (C) preparing the grasp, (D) grasping the screwdriver, (E) letting the operator move the tool, (F) letting the operator screw, (E) letting the operator move the tool, (F) letting the operator screw, (A) going to initial pose, (B) releasing the tool.

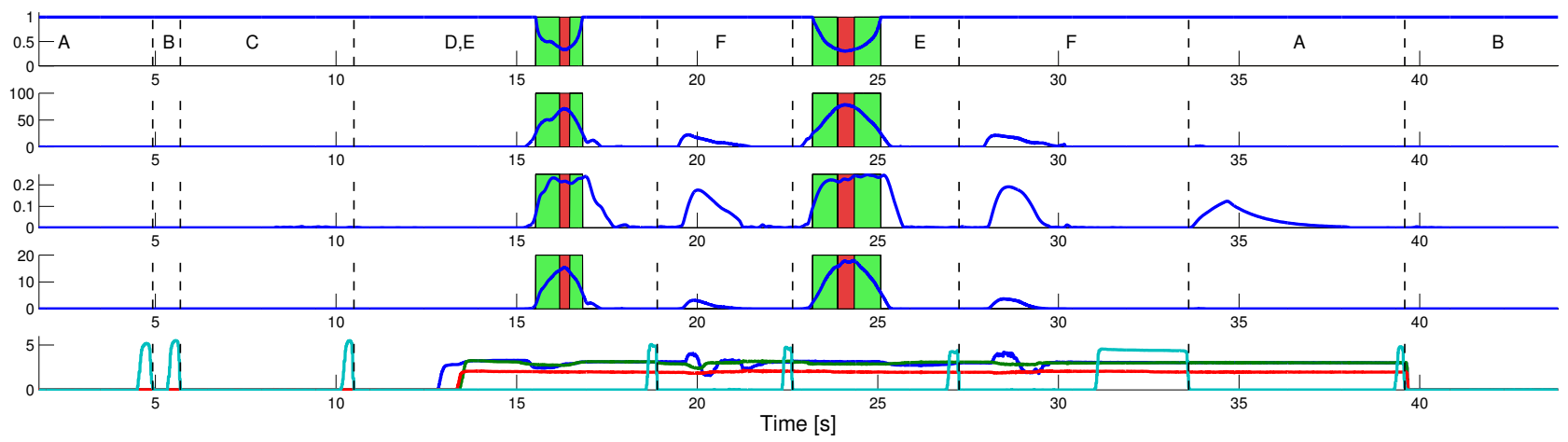

Fig. 4: Experimental results. From top to bottom: damping scaling factor $K$, external force magnitude $|\mathbf{f}|(\mathrm{N})$, tool velocity $|\mathbf{v}|(\mathrm{m} / \mathrm{s})$, tool power $P(\mathrm{~W})$, Fingertip contact forces (index finger in blue, ring finger in green, little finger in red and thumb in cyan) $(\mathrm{N})$. The green and red areas represent velocity $\left(K_{v}\right)$ and power $\left(K_{P}\right)$ adaptive limitations, respectively. The vertical dashed lines delimit the states.

\section{ACKNOWLEDGMENTS}

This work has been supported by the ANR (French National Research Agency) SISCob project ANR-14-CE27-0016.

\section{REFERENCES}

[1] R. Alami, et al. Safe and dependable physical human-robot interaction in anthropic domains: State of the art and challenges. In IEEE/RSJ Int. Conf. on Intelligent Robots and Systems, IROS, 2006.

[2] S. Haddadin, A. Albu-Schäffer, and G. Hirzinger. Requirements for safe robots: Measurements, analysis and new insights. Int. Journal of Robotics Research, IJRR, 2009.

[3] A. De Luca and F. Flacco. Integrated control for pHRI: collision avoidance, detection, reaction and collaboration. In IEEE RAS/EMBS Int. Conf. on Biomedical Robotics and Biomechatronics, BIOROB, 2012.

[4] J. Krüger, T.K. Lien, and A. Verl. Cooperation of human and machines in assembly lines. CIRP Annals - Manufacturing Technology, 58(2):628 - 646, 2009.

[5] ISO 10218-1:2011 Robot for industrial environments - Safety requirements - Part 1 : Robot. Technical report, International Organization for Standardization, Geneva, Switzerland, 2006.

[6] Robotiq. Collaborative robot ebook - http://blog.robotiq.com.

[7] M. Zinn, O. Khatib, B. Roth, and J.K. Salisbury. Playing it safe. IEEE Robotics and Automation Magazine, 11(2):12-21, 2004.

[8] A. Albu-Schaffer et al. Soft robotics. IEEE Robotics and Automation Magazine, 15(3):20-30, 2008.

[9] R. Schiavi, G. Grioli, S. Sen, and A. Bicchi. VSA-II: A novel prototype of variable stiffness actuator for safe and performing robots interacting with humans. In IEEE Int. Conf. on Robotics and Automation ICRA, 2008.
[10] N. Hogan. Impedance control: An approach to manipulation: Part II-Implementation. Journal of Dynamic Systems, Measurement, and Control, 107(1):8-16, 1985.

[11] S. Jung, T.C. Hsia, and R.G. Bonitz. Force tracking impedance control of robot manipulators under unknown environment. IEEE Trans. on Control Systems Technology, 12(3):474-483, May 2004.

[12] F. Almeida, A. Lopes, and P. Abreu. Force-impedance control: A new control strategy of robotic manipulators. In Recent Advances in Mechatronics, Springer, Singapore, pages 126-137, 1999.

[13] V. Duchaine and C. Gosselin. Safe, stable and intuitive control for physical human-robot interaction. In IEEE Int. Conf. on Robotics and Automation, ICRA, pages 3383-3388, 2009.

[14] H. Sadeghian, L. Villani, M. Keshmiri, and B. Siciliano. Experimental study on task space control during physical human robot interaction. In 2nd RSI/ISM Int. Conf. on Robotics and Mechatronics (ICRoM), pages 125-130, Oct 2014.

[15] A Vick, D Surdilovic, and J. Krüger. Safe physical human-robot interaction with industrial dual-arm robots. In 9th IEEE Workshop on Robot Motion and Control (RoMoCo), pages 264-269, 2013.

[16] G. Muscio, F. Pierri, and J. Trinkle. A hand/arm controller that simultaneously regulates internal grasp forces and the impedance of contacts with the environment. In IEEE Int. Conf. on Robotics and Automation, ICRA, pages 895-900, May 2014.

[17] B. Navarro, P. Kumar, A. Fonte, P. Fraisse, G. Poisson, and A. Cherubini. Active calibration of tactile sensors mounted on a robotic hand. In IEEE/RSJ IROS Workshop on Multimodal sensor-based robot control for HRI and soft manipulation, 2015.

[18] V. Ciobanu, A. Petrescu, N. Hendrich, and J. Zhang. Tactile sensor value preprocessing pipeline. In 17th Int. Conf. on System Theory, Control and Computing (ICSTCC), pages 674-680, Oct 2013. 